\title{
Periodic Solutions of a Class of Second-Order Differential Equation
}

\author{
Zeyneb Bouderbala1', Jaume Llibre², Amar Makhlouf ${ }^{1}$ \\ ${ }^{1}$ Department of Mathematics, University of Annaba, Elhadjar, Annaba, Algeria \\ ${ }^{2}$ Departament de Matemàtiques, Universitat Autònoma de Barcelona, Barcelona, Spain \\ Email: zeynebbouderbala@yahoo.fr,jllibre@mat.uab.cat,makhloufamar@yahoo.fr
}

Received 25 December 2015; accepted 26 February 2016; published 29 February 2016

Copyright (C) 2016 by authors and Scientific Research Publishing Inc.

This work is licensed under the Creative Commons Attribution International License (CC BY).

http://creativecommons.org/licenses/by/4.0/

(c) (†) Open Access

\section{Abstract}

We study the periodic solutions of the second-order differential equations of the form

$$
\ddot{x}+3 x \dot{x}+x^{3}+F(t)\left(\dot{x}+x^{2}\right)+G(t) x+H(t)=0,
$$

where the functions $F(t), G(t)$ and $H(t)$ are periodic of period $2 \pi$ in the variable $t$.

\section{Keywords}

\section{Periodic Solution, Differential Equation, Averaging Theory}

\section{Introduction and Statement of the Main Results}

In this paper we shall study the existence of periodic solutions of the second-order differential equation of the form

$$
\ddot{x}+3 x \dot{x}+x^{3}+F(t)\left(\dot{x}+x^{2}\right)+G(t) x+H(t)=0,
$$

where the dot denotes derivative with respect to the time $t$, and the functions $F(t), G(t)$ and $H(t)$ are periodic of period $2 \pi$ in the variable $t$.

We note that the second-order differential Equation (1), when $F=G=H=0$, appears in the Ince's catalog of equations possessing the Painlevé property (see [1]). Moreover, the differential equation $\ddot{x}+3 x \dot{x}+x^{3}=0$ is well known in many areas of mathematics and physics, and it possesses the algebra $s l(3, \mathbb{R})$ of Lie point symmetries (see for more details in the paper [2] and the references quoted there).

In a recent paper [3] (see also [4] [5]), the second-order differential Equation (1) has been studied when $F=H=0$. A study of coupled quadratic unharmonic oscillators in terms of the Painlevé analysis and inte- 
grability can be seen in [6], and studies on the second-order differential equations can be seen in [7]. Other approach to the periodic solutions of second-order differential equations can be found in [8].

Here we study the periodic solutions of the second-order differential Equation (1) when $F(t)=\varepsilon f(t)$, $G(t)=1+\varepsilon g(t)$, and $H(t)=\varepsilon^{k} h(t)$ with $k=1,2$. Our main results are the following ones.

Theorem 1. We define the functions

$$
\begin{aligned}
& \mathcal{F}_{1}\left(X_{0}, Y_{0}\right)=-\int_{0}^{2 \pi} F\left(t, X_{0}, Y_{0}\right) \sin t \mathrm{~d} t, \\
& \mathcal{F}_{2}\left(X_{0}, Y_{0}\right)=\int_{0}^{2 \pi} F\left(t, X_{0}, Y_{0}\right) \cos t \mathrm{~d} t
\end{aligned}
$$

where

$$
\begin{gathered}
F\left(t, X_{0}, Y_{0}\right)=-h(t)-g(t) A(t)-f(t) B(t)-3 A(t) B(t), \\
A(t)=X_{0} \cos t+Y_{0} \sin t, \\
B(t)=-X_{0} \sin t+Y_{0} \cos t .
\end{gathered}
$$

Assume that the functions $F(t)=\varepsilon f(t), G(t)=1+\varepsilon g(t)$ and $H(t)=\varepsilon^{2} h(t)$ are $2 \pi$-periodic. Then for $\varepsilon \neq 0$ sufficiently small and for every $\left(X_{0}^{*}, Y_{0}^{*}\right)$ solution of the system $\mathcal{F}_{j}\left(X_{0}, Y_{0}\right)=0$ for $j=1,2$, satisfy

$$
\left.\operatorname{det}\left(\frac{\partial\left(\mathcal{F}_{1}, \mathcal{F}_{2}\right)}{\partial\left(X_{0}, Y_{0}\right)}\right)\right|_{\left(X_{0}, Y_{0}\right)=\left(x_{0}^{*}, Y_{0}^{*}\right)} \neq 0,
$$

the differential Equation (1) has a $2 \pi$-periodic solution $x(t, \varepsilon)=\varepsilon\left(X_{0}^{*} \cos t+Y_{0}^{*} \sin t\right)+O\left(\varepsilon^{2}\right)$.

Theorem 1 is proved in section 3 using the averaging theory described in section 2 . Two applications of Theorem 1 are the following.

Corollary 1. We consider the differential Equation (1) with $F(t)=\varepsilon\left(1-\cos ^{2} t\right), G(t)=1+\varepsilon \sin ^{2} t$ and $H(t)=\varepsilon^{2} \sin t$. Then for $\varepsilon \neq 0$ sufficiently small, this differential equation has a $2 \pi$-periodic solution $x(t, \varepsilon)=\varepsilon 2(\sin t-\cos t) / 3+O\left(\varepsilon^{2}\right)$.

Corollary 2. We consider the differential Equation (1) with $F(t)=\varepsilon\left(1-\cos ^{2} t+2 \cos ^{4} t\right)$, $G(t)=1+\varepsilon\left(\sin ^{2} t+2 \sin ^{4} t\right)$ and $H(t)=\varepsilon^{2}\left(\sin t+\sin ^{3} t\right)$. Then for $\varepsilon \neq 0$ sufficiently small, this differential equation has a $2 \pi$-periodic solution $x(t, \varepsilon)=\varepsilon(21 \cos t-7 \sin t) / 20+O\left(\varepsilon^{2}\right)$.

Corollaries 1 and 2 are also proved in section 3.

Theorem 2. Assuming that

$$
\int_{0}^{2 \pi} h(t) \sin t \mathrm{~d} t=0, \quad \int_{0}^{2 \pi} h(t) \cos t \mathrm{~d} t=0,
$$

and setting

$$
\begin{aligned}
& \mathcal{F}_{1}\left(X_{0}, Y_{0}\right)=-\int_{0}^{2 \pi} f\left(t, X_{0}, Y_{0}\right) \sin t \mathrm{~d} t, \\
& \mathcal{F}_{2}\left(X_{0}, Y_{0}\right)=\int_{0}^{2 \pi} f\left(t, X_{0}, Y_{0}\right) \cos t \mathrm{~d} t
\end{aligned}
$$

with

$$
\begin{gathered}
f\left(t, X_{0}, Y_{0}\right)=-g(t) A(t)-f(t) B(t)-3 A(t) B(t), \\
A(t)=X_{0} \cos t+Y_{0} \sin t-\int_{0}^{t} h(\tau) \sin (t-\tau) \mathrm{d} \tau, \\
B(t)=-X_{0} \sin t+Y_{0} \cos t-\int_{0}^{t} h(\tau) \cos (t-\tau) \mathrm{d} \tau .
\end{gathered}
$$

Assume that $F(t)=\varepsilon f(t), G(t)=1+\varepsilon g(t)$ and $H(t)=\varepsilon h(t)$ are $2 \pi$-periodic functions. Then for $\varepsilon \neq 0$ sufficiently small and for every $\left(X_{0}^{*}, Y_{0}^{*}\right)$ solution of the system $\mathcal{F}_{j}\left(X_{0}, Y_{0}\right)=0$ for $j=1,2$ satisfy (3), the differential Equation (1) has a periodic solution 


$$
x(t, \varepsilon)=\varepsilon\left(X_{0}^{*} \cos t+Y_{0}^{*} \sin t-\int_{0}^{t} h(\tau) \sin (t-\tau) \mathrm{d} \tau\right)+O\left(\varepsilon^{2}\right) .
$$

Theorem 2 is proved in section 4 . Two applications of Theorem 2 are the following.

Corollary 3. We consider the differential Equation (1) with $F(t)=\varepsilon(\sin (2 t)+\cos (2 t)), G(t)=1+\varepsilon \sin t$ and $H(t)=\varepsilon 2 \cos ^{2} t$. Then for $\varepsilon \neq 0$ sufficiently small, this differential equation has a $2 \pi$-periodic solution

$$
x(t, \varepsilon)=\varepsilon\left((-2 \cos t+15 \sin t) / 31+2 \cos ^{2} t(\cos t-1)\right)+O\left(\varepsilon^{2}\right) .
$$

Corollary 4. We consider the differential Equation (1) with $F(t)=\varepsilon \sin t, G(t)=1+\varepsilon \sin ^{2} t$ and $H(t)=\varepsilon 2 \cos (2 t)$. Then for $\varepsilon \neq 0$ sufficiently small, this differential equation has a periodic solution

$$
x(t, \varepsilon)=\varepsilon\left(2(\cos t-1) \cos (2 t)-\frac{8}{5} \sin t\right)+O\left(\varepsilon^{2}\right) .
$$

Corollaries 3 and 4 are also proved in section 4 .

\section{Basic Results on Averaging Theory}

We state the results from the averaging method that we shall use for proving the results of this work.

We consider differential systems of the form

$$
\boldsymbol{x}^{\prime}=F_{0}(t, \boldsymbol{x})+\varepsilon F_{1}(t, \boldsymbol{x})+\varepsilon^{2} F_{2}(t, \boldsymbol{x}, \varepsilon),
$$

where $\varepsilon$ is a small parameter, and the functions $F_{0}, F_{1}: \mathbb{R} \times \Omega \rightarrow \mathbb{R}^{n}$ and $F_{2}: \mathbb{R} \times \Omega \times\left(-\varepsilon_{0}, \varepsilon_{0}\right) \rightarrow \mathbb{R}^{n}$ are $\mathcal{C}^{2}$ functions, $T$-periodic in the variable $t$, and $\Omega$ is an open subset of $\mathbb{R}^{n}$. Suppose that the unperturbed system

$$
\boldsymbol{x}^{\prime}=F_{0}(t, \boldsymbol{x}),
$$

has a submanifold of dimension $n$ of $T$-periodic solutions, i.e. of periodic solutions of period $T$.

We denote by $\boldsymbol{x}(t, \boldsymbol{z}, 0)$ the solution of system (6) such that $\boldsymbol{x}(0, \boldsymbol{z}, 0)=\mathbf{z}$. We consider the first variational equation of system (6) on the periodic solution $x(t, \mathbf{z}, 0)$, i.e.

$$
\boldsymbol{y}^{\prime}=D_{x} F_{0}(t, x(t, \mathbf{z}, 0)) \boldsymbol{y}
$$

where $y$ is an $n \times n$ matrix. Let $M_{z}(t)$ the fundamental matrix of system (7) such that $M_{z}(0)$ is the identity matrix of $\mathbb{R}^{n}$.

By assumption there exists an open set $V$ such that $\mathrm{Cl}(V) \subset \Omega$ and for each $\mathbf{z} \in \mathrm{Cl}(V), \boldsymbol{x}(t, \mathbf{z}, 0)$ is $T$-periodic. Therefore we have the following result.

Theorem 3. We suppose that there is an open and bounded set $V$ with $\mathrm{Cl}(V) \subset \Omega$ such that for each $\mathbf{z} \in \mathrm{Cl}(V)$, the solution $\mathbf{x}(t, \mathbf{z}, 0)$ is T-periodic, and let $\mathcal{F}: \mathrm{Cl}(V) \rightarrow \mathbb{R}^{n}$ be the function defined by

$$
\mathcal{F}(\mathbf{z})=\int_{0}^{T} M_{\mathbf{z}}^{-1}(t) F_{1}(t, x(t, \mathbf{z}, 0)) \mathrm{d} t .
$$

If there is $\alpha \in V$ with $\mathcal{F}(\alpha)=0$ and $\operatorname{det}((\mathrm{d} \mathcal{F} / \mathrm{d} \boldsymbol{z})(\alpha)) \neq 0$, then there is a $T$-periodic solution $\boldsymbol{x}(t, \varepsilon)$ of system (5) satisfying $\boldsymbol{x}(t, \varepsilon)=\boldsymbol{x}(t, \mathbf{z}, 0)+O(\varepsilon)$.

Theorem 3 is due to Malkin [9] and Roseau [10], for a new and shorter proof (see [11]).

\section{Proof of Theorem 1 and Its Two Corollaries}

Proof of Theorem 1. Introducing the variable $y=\dot{x}$, we can write the second-order differential Equation (1) as the following first-order differential system

$$
\begin{aligned}
& \dot{x}=y, \\
& \dot{y}=-3 x y-x^{3}-F(t)\left(y+x^{2}\right)-G(t) x-H(t) .
\end{aligned}
$$

Doing the rescaling $(x, y)=(\varepsilon X, \varepsilon Y)$, we obtain the system 


$$
\begin{aligned}
& \dot{X}=Y \\
& \dot{Y}=-X+\varepsilon(-h(t)-g(t) X-f(t) Y-3 X Y)+\varepsilon^{2}\left(-f(t) X^{2}-X^{3}\right) .
\end{aligned}
$$

System (10) with $\varepsilon=0$ is the unperturbed system, otherwise system (10) is the perturbed system. The unperturbed system has a unique singular point, the origin of coordinates. The solution $(X(t), Y(t))$ of the unperturbed system such that $(X(0), Y(0))=\left(X_{0}, Y_{0}\right)$ is

$$
X(t)=X_{0} \cos t+Y_{0} \sin t, \quad Y(t)=-X_{0} \sin t+Y_{0} \cos t .
$$

Note that all these periodic orbits have period $2 \pi$. Using the notation introduced in section 2 . We have that $\boldsymbol{x}=(X, Y), \quad \boldsymbol{z}=\left(X_{0}, Y_{0}\right), \quad F_{0}(x, t)=(Y,-X), \quad F_{1}(x, t)=(0,-h(t)-g(t) X-f(t) Y-3 X Y)$ and $F_{2}(x, t)=\left(0,-f(t) X^{2}-X^{3}\right)$.

The fundamental matrix solution $M_{z}(t)$ is independent of the initial condition $\mathbf{z}$, and denoting it by $M(t)$ we obtain

$$
M(t)=\left(\begin{array}{cc}
\cos t & \sin t \\
-\sin t & \cos t
\end{array}\right)
$$

Now we compute the function $\mathcal{F}(\mathbf{z})=\left(\mathcal{F}_{1}\left(X_{0}, Y_{0}\right), \mathcal{F}_{2}\left(X_{0}, Y_{0}\right)\right)$ given in (8), and we get the functions (2) of the statement of Theorem 1.

By Theorem 3 each zero $\left(X_{0}^{*}, Y_{0}^{*}\right)$ of system $\mathcal{F}_{1}\left(X_{0}, Y_{0}\right)=\mathcal{F}_{2}\left(X_{0}, Y_{0}\right)=0$ satisfying (3), provides a $2 \pi$ periodic solution $(X(t, \varepsilon), Y(t, \varepsilon))$ of system (10) with $\varepsilon \neq 0$ sufficiently small such that

$$
(X(t, \varepsilon), Y(t, \varepsilon))=\left(X_{0}^{*} \cos t+Y_{0}^{*} \sin t,-X_{0}^{*} \sin t+Y_{0}^{*} \cos t\right)+O(\varepsilon) .
$$

Going back through the change of variables for every periodic solution $(X(t, \varepsilon), Y(t, \varepsilon))$ of system (10) with $\varepsilon \neq 0$ sufficiently small, we obtain a $2 \pi$-periodic solution $x(t, \varepsilon)=\varepsilon\left(X_{0}^{*} \cos t+Y_{0}^{*} \sin t\right)+O\left(\varepsilon^{2}\right)$ of the differential Equation (1) with $\varepsilon \neq 0$ sufficiently small. This completes the proof of Theorem 1 .

Proof of Corollary 1. We must apply Theorem 1 with

$$
f(t)=1-\cos ^{2} t, \quad g(t)=\sin ^{2} t, \quad h(t)=\sin t .
$$

We compute the functions $\mathcal{F}_{1}$ and $\mathcal{F}_{2}$ of the statement of Theorem 1 , and we obtain

$$
\mathcal{F}_{1}\left(X_{0}, Y_{0}\right)=\frac{\pi}{4}\left(4-3 X_{0}+3 Y_{0}\right), \quad \mathcal{F}_{2}\left(X_{0}, Y_{0}\right)=\frac{\pi}{4}\left(-X_{0}-Y_{0}\right)
$$

System $\mathcal{F}_{1}=\mathcal{F}_{2}=0$ has the zero $\left(X_{0}^{*}, Y_{0}^{*}\right)=(2 / 3,-2 / 3)$. Since the Jacobian (3) at this zero is $3 \pi^{2} / 8$, we obtain using Theorem 1 the periodic solution given in the statement of the corollary.

Proof of Corollary 2. We apply Theorem 1 with

$$
f(t)=1-\cos ^{2} t+2 \cos ^{4} t, \quad g(t)=\sin ^{2} t+2 \sin ^{4} t, \quad h(t)=\sin t+\sin ^{3} t .
$$

Computing the functions $\mathcal{F}_{1}$ and $\mathcal{F}_{2}$ of Theorem 1 we get

$$
\mathcal{F}_{1}\left(X_{0}, Y_{0}\right)=\frac{\pi}{4}\left(7-4 X_{0}+8 Y_{0}\right), \quad \mathcal{F}_{2}\left(X_{0}, Y_{0}\right)=-\frac{\pi}{2}\left(X_{0}+3 Y_{0}\right) .
$$

System $\mathcal{F}_{1}=\mathcal{F}_{2}=0$ has the zero $\left(X_{0}^{*}, Y_{0}^{*}\right)=(21 / 20,-7 / 20)$. Since the Jacobian (3) at this zero is $5 \pi^{2} / 2$ the corollary follows.

\section{Proof of Theorem 2 and Its Corollaries}

Proof of Theorem 2. As in the proof of Theorem 1, the second-order differential Equation (1) can be written as the first order differential system (9). Doing the rescaling $(x, y)=(\varepsilon X, \varepsilon Y)$, we obtain the system

$$
\begin{aligned}
& \dot{X}=Y \\
& \dot{y}=-X-h(t)+\varepsilon(-g(t) X-f(t) Y-3 X Y)+\varepsilon^{2}\left(-f(t) X^{2}-X^{3}\right) .
\end{aligned}
$$


System (11) with $\varepsilon=0$ is the unperturbed system, otherwise it is the perturbed system.

The solution $(X(t), Y(t))$ of the unperturbed system such that $(X(0), Y(0))=\left(X_{0}, Y_{0}\right)$ is

$$
\begin{aligned}
& X(t)=X_{0} \cos t+Y_{0} \sin t-\int_{0}^{t} h(\tau) \sin (t-\tau) \mathrm{d} \tau, \\
& Y(t)=-X_{0} \sin t+Y_{0} \cos t-\int_{0}^{t} h(\tau) \cos (t-\tau) \mathrm{d} \tau .
\end{aligned}
$$

Note that these periodic orbits have period $2 \pi$. Using the notation introduced in section 2 . We have that $\boldsymbol{x}=(X, Y), \quad \boldsymbol{z}=\left(X_{0}, Y_{0}\right), \quad F_{0}(\boldsymbol{x}, t)=(Y,-X-h), \quad F_{1}(\boldsymbol{x}, t)=(0,-g(t) X-f(t) Y-3 X Y)$ and $F_{2}(x, t)=\left(0,-f(t) X^{2}-X^{3}\right)$.

The fundamental matrix solution $M_{z}(t)$ is independent of the initial condition $\mathbf{z}$ and it is

$$
M(t)=\left(\begin{array}{cc}
\cos t & \sin t \\
-\sin t & \cos t
\end{array}\right)
$$

We compute the function $\mathcal{F}(\mathbf{z})=\left(\mathcal{F}_{1}\left(X_{0}, Y_{0}\right), \mathcal{F}_{2}\left(X_{0}, Y_{0}\right)\right)$ given in (8), and we get the functions (4) of the statement of Theorem 2.

By Theorem 3, each zero $\left(X_{0}^{*}, Y_{0}^{*}\right)$ of system $\mathcal{F}_{1}\left(X_{0}, Y_{0}\right)=\mathcal{F}_{2}\left(X_{0}, Y_{0}\right)=0$ satisfying (3), provides a $2 \pi$ periodic solution $(X(t, \varepsilon), Y(t, \varepsilon))$ of system (11) with $\varepsilon \neq 0$ sufficiently small such that

$$
\left(\begin{array}{l}
X(t, \varepsilon) \\
Y(t, \varepsilon)
\end{array}\right)=\left(\begin{array}{c}
X_{0}^{*} \cos t+Y_{0}^{*} \sin t-\int_{0}^{t} h(\tau) \sin (t-\tau) \mathrm{d} \tau \\
-X_{0}^{*} \sin t+Y_{0}^{*} \cos t-\int_{0}^{t} h(\tau) \cos (t-\tau) \mathrm{d} \tau
\end{array}\right)+O(\varepsilon) .
$$

Going back through the change of variables for every periodic solution $(X(t, \varepsilon), Y(t, \varepsilon))$ of system (11) with $\varepsilon \neq 0$ sufficiently small, we obtain a $2 \pi$-periodic solution

$$
x(t, \varepsilon)=\varepsilon\left(X_{0}^{*} \cos t+Y_{0}^{*} \sin t-\int_{0}^{t} h(\tau) \sin (t-\tau) \mathrm{d} \tau\right)+O\left(\varepsilon^{2}\right)
$$

of the differential Equation (1) for $\varepsilon \neq 0$ sufficiently small. This completes the proof of Theorem 2.

Proof of Corollary 3. We apply Theorem 2 with

$$
f(t)=\sin (2 t)+\cos (2 t), \quad g(t)=\sin t, \quad h(t)=2 \cos ^{2} t
$$

We compute the functions $\mathcal{F}_{1}$ and $\mathcal{F}_{2}$ of the statement of Theorem 2, and we obtain

$$
\mathcal{F}_{1}\left(X_{0}, Y_{0}\right)=\frac{\pi}{2}\left(2+X_{0}-4 Y_{0}\right), \quad \mathcal{F}_{2}\left(X_{0}, Y_{0}\right)=\frac{\pi}{2}\left(1+8 X_{0}-Y_{0}\right)
$$

System $\mathcal{F}_{1}=\mathcal{F}_{2}=0$ has the solution $\left(X_{0}^{*}, Y_{0}^{*}\right)=(-2 / 31,15 / 31)$. Since the Jacobian (3) is $31 \pi^{2} / 4$, by Theorem 2 we obtain the periodic solution of the statement of the corollary.

Proof of Corollaryc 4. We apply Theorem 2 with

$$
f(t)=\sin t, \quad g(t)=\sin ^{2} t, \quad h(t)=2 \cos (2 t) .
$$

We compute the functions $\mathcal{F}_{1}$ and $\mathcal{F}_{2}$ of the statement of Theorem 2, and we obtain

$$
\mathcal{F}_{1}\left(X_{0}, Y_{0}\right)=\frac{3 \pi}{4}\left(8+5 Y_{0}\right), \quad \mathcal{F}_{2}\left(X_{0}, Y_{0}\right)=\frac{11 \pi}{4} X_{0} .
$$

System $\mathcal{F}_{1}=\mathcal{F}_{2}=0$ has the solution $\left(X_{0}^{*}, Y_{0}^{*}\right)=(0,-8 / 5)$. Since the Jacobian (3) is $-165 \pi^{2} / 16$, by Theorem 2 we obtain the periodic solution of the statement of the corollary.

\section{Acknowledgements}

The second author is partially supported by a MINECO grant MTM2013-40998-P, an AGAUR grant number 2014SGR568, and the grants FP7-PEOPLE-2012-IRSES 318999 and 316338. 


\section{References}

[1] Ince, E.L. (1927) Ordinary Differential Equations. Longmans, London, 1927.

[2] Karasu, A. and Leach, P.G.L. (2009) Nonlocal Symmetries and Integrable Ordinary Differential Equations: $\ddot{x}+3 x \dot{x}+x^{3}=0$ and Its Genralizations. Journal of Mathematical Physics, 50, Article ID: 073509, 17 p.

[3] Chandrasekar, V.K., Senthilvelan, M. and Lakshmanan, M. (2005) Lienard-Type Nonlinear Oscillator. Physical Review E, 72, Article ID: 066203, 8 p.

[4] Chandrasekar, V.K., Senthilvelan, M. and Lakshmanan, M. (2012) A Systematic Method of Finding Linearizing Transformations for Nonlinear Ordinary Differential Equations: I. Scalar Case. Journal of Nonlinear Mathematical Physics, 19, Article ID: 1250012, 21 p.

[5] Chandrasekar, V.K., Senthilvelan, V.K. and Lakshmanan, M. (2012) A Systematic Method of Finding Linearizing Transformations for Nonlinear Ordinary Differential Equations: II. Extension to Coupled ODEs. Journal of Nonlinear Mathematical Physics, 19, Article ID: 1250013, 23 p.

[6] Lakshmanan, M. and Sahadevan, R. (1985) Coupled Quadratic Anharmonic Oscillators, Painlevé Analysis and Integrability. Physical Review A, 31, 861-876. http://dx.doi.org/10.1103/PhysRevA.31.861

[7] Ferreira, C., López, J.L. and Pérez, S. (2014) Ester Convergent and Asymptotic Expansions of Solutions of SecondOrder Differential Equations with a Large Parameter. Analysis and Applications, 12, 523-536. http://dx.doi.org/10.1142/S0219530514500328

[8] Li, J., Luo, J. and Wang, Z. (2014) Periodic Solutions of Second Order Impulsive Differential Equations at Resonance via Variational Approach. Mathematical Modelling and Analysis, 19, 664-675. http://dx.doi.org/10.3846/13926292.2014.980864

[9] Malkin, I.G. (1956) Some Problems of the Theory of Nonlinear Oscillations. Gosudarstv. Izdat. Tehn-Teor. Lit., Moscow. (In Russian).

[10] Roseau, M. (1985) Vibrations non linéaires et théorie de la stabilité. Springer Tracts in Natural Philosophy, Vol. 8, Springer, New York, 1985.

[11] Buica, A., Françoise, J.P. and Llibre, J. (2007) Periodic Solutions of Nonlinear Periodic Differential Systems with a Small Parameter. Communications on Pure and Applied Analysis, 6, 103-111. 